\title{
Universal Scaling Laws of Hydraulic Fracturing
}

\author{
Bo-Hua Sun \\ School of Civil Engineering 8 Institute of Mechanics and Technology \\ $X i$ 'an University of Architecture and Technology, \\ $X i$ 'an 710055, China \\ email: sunbohua@xauat.edu.cn
}

\begin{abstract}
The hydraulic fracturing is studied by using dimensional analysis. A universal scaling law of the hydraulic fracturing, $\left(p+\frac{1}{2} \rho U^{2}\right) \sqrt{\pi \ell}=K_{C}$, is obtained. This simple relation has not been seen in the literature.
\end{abstract}

Hydraulic fracturing, as shown in Fig. 1, is a wellstimulation technique in which rock is fractured by a pressurized liquid $[1,2]$.

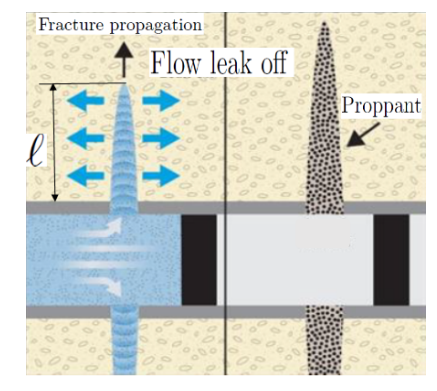

FIG. 1: Hydraulic fracturing: perforation, fracture propagation, flow leak off and proppant holding

The whole fracking process can be viewed as an interaction between high pressure flow and rock, the flow acts as a spear and rock as a shield, they fight each other. The flow make the rock instable and rock resist it to keep itself stable. Delicate dynamical balance or equivalence of between the flow and rock is vital for the hydraulic fracturing process, therefore, the process must be controlled by certain kind scaling laws. There are number of numerical investigations on the topic. However, there is no any general discussion on the scaling law of the process. The aim of this short paper is to apply dimensional analysis to formulate a universal scaling law of the hydraulic fracturing $[1,2]$.

In the process of hydraulic fracturing, if we omit temperature influence, the process is controlled by following variables. The density of fracking fluid $\rho$, kinematic viscosity of fracking fluid $\nu$, crustal stress $p$, flow velocity $U$, rock fracture stiffness $K_{C}$ and characteristic length of crack $\ell$. Fracturing rocks at great depth frequently becomes suppressed by pressure, this suppression process is particularly significant in "tensile" (Mode I) fractures which require the walls of the fracture to move against this pressure, so $K_{C}$ can be chosen as $K_{C}=K_{I}$ of mode I. The dimensions of variables can be listed in the following table.

TABLE I: Hydraulic fracturing dimensions

\begin{tabular}{cccccc}
\hline$\rho$ & $\nu$ & $p$ & $U$ & $K_{C}$ & $\ell$ \\
\hline$M L^{-3}$ & $L^{2} T^{-1}$ & $L^{-1} M T^{-2}$ & $L T^{-1}$ & $L^{-1 / 2} M T^{-2}$ & $L$ \\
\hline
\end{tabular}

Since the Reynolds number in the hydraulic fracture process is small, so the influence of viscosity of fracking fluid can be omitted. Therefore, it is clear that the crack process must have a relation as follows $[4,5]$

$$
f\left(\ell, P, K_{C}\right)=0,
$$

where the total stress or pressure is $P=p+p_{d y m}$, and dynamical flow pressure $p_{d y m}=\frac{1}{2} \rho U^{2}$.

There are three variables in equation 1 . Since it is a mechanics system, which has three basic unit such as time $T$, length $L$ and mass $M$. Then from Buckingham's $\Pi$ theorem $[4,5]$, we can construct only one dimensionless parameter $\Pi$.

The $\Pi$ can be universally expressed as $\Pi=P \ell^{a} K_{c}^{b}$, whose dimension is

$$
\operatorname{dim}[\Pi]=\operatorname{dim}\left[P \ell^{a} K_{c}^{b}\right]=L^{-1+a-b / 2} M^{1+b} T^{-2-2 b},
$$

where $a, b$ are to to determined constants. From $1+b=$ $0,-1+a-b / 2=0$ and $-2-2 b=0$, we can determine $a=-1 / 2$ and $b=-1$. Hence we obtain five $\Pi$ as follows

$$
\Pi=P \ell^{1 / 2} K_{C}^{-1}=\left(p+\frac{1}{2} \rho U^{2}\right) \ell^{1 / 2} K_{C}^{-1} .
$$

Because we have only one $\Pi$, and according to Buckingham's $\Pi$ theorem $[4,5]$, this only $\Pi$ must be a constant, namely

$$
\Pi=\left(p+\frac{1}{2} \rho U^{2}\right) \ell^{1 / 2} K_{C}^{-1}=\text { constant } .
$$

The problem is how to determine the constant.

If there were no flow pressure, namely $U=0$, the above relation is reduced to $p \ell^{1 / 2} K_{C}^{-1}=$ constant.. For this problem, the linear classical fracture theory gives $\sigma \sqrt{\pi \ell}=K_{c}$, where $\sigma$ is fracture stress. By simple comparison, we can obtain the constant as follows

$$
\text { constant }=\frac{1}{\sqrt{\pi}} \text {. }
$$

Substituting the constant value back to the equation 4 , we have

$$
\left(p+\frac{1}{2} \rho U^{2}\right) \sqrt{\pi \ell}=K_{C}
$$

This is the universal scaling law for hydraulic fracture as shown in Fig. 2 below. 


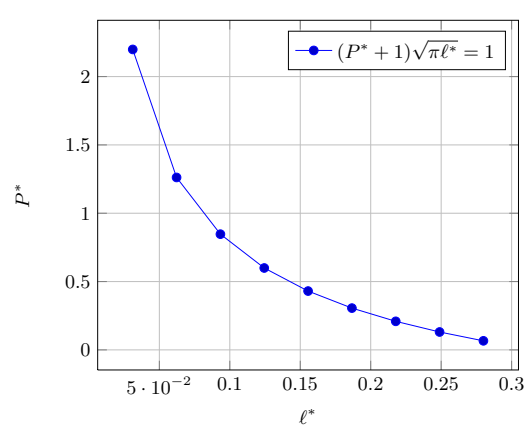

FIG. 2: The fracture flow speed vs. crack length, where $P^{*}=$ $\frac{2 p}{\rho U^{2}}, \ell^{*}=\ell \frac{\rho U^{2}}{2 K_{C}}$

If the dynamical part, $\rho U^{2} / 2$ of the pressure is greater than the crustal stress, $p$, then the formula (6) is reduced to $\frac{1}{2} \rho U^{2} \sqrt{\pi \ell}=K_{C}$. Hence the fracture flow critical speed can be predicted as follows

$$
U \approx(\pi \ell)^{-1 / 4} \sqrt{\frac{2 K_{C}}{\rho}} .
$$

This scaling law reveals that the hydro crack flow speed is proportional to -4 power law of crack length $\ell$, as shown in Fig. 3 below. and the crack length vs. fracture flow

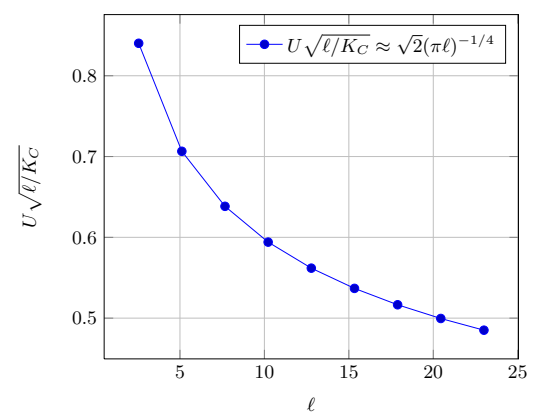

FIG. 3: The fracture flow speed vs. crack length

speed as shown in Fig. 4 below.

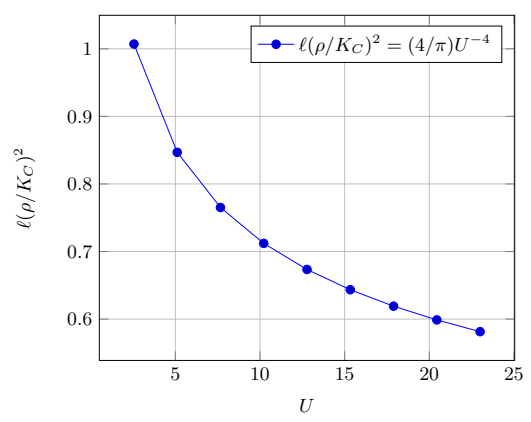

FIG. 4: The crack length vs. fracture flow speed

It must noted that rock fracture would be happening in a time. Impact under high pressure, the development from micro cracking, growth, interconnecting to a macro crack will needs a time process, in other words, the rock has an characteristic fracture time $t_{p}$, with time dimension $T$.

Having concern the speed of energy wave in the rock, defined by $\sqrt{\frac{p}{\rho}}$, if set $\ell \sqrt{\frac{\rho}{p}}=t_{c}$ as fracturing time of the rock, then $t_{c} / t_{p}$ is the Deborah number $D e$. The rock is softer or stronger as the Deborah number is getting smaller or bigger, respectively.

Therefore the formula (6) can be extended into

$$
\left(p+\frac{1}{2} \rho U^{2}\right) \sqrt{\pi \ell}=C(D e) K_{C}
$$

where $C(D e)$ is an undetermined function of the Deborah number $D e$, which should be determined by other methods. If just take its linear approximation, namely $C(D e) \approx \alpha \frac{\ell}{t_{p}} \sqrt{\frac{\rho}{p}}$, where $\alpha$ is a constant to be confirmed.

In summary, this short article obtained a universal scaling law for hydraulic fracturing: $\left(p+\frac{1}{2} \rho U^{2}\right) \sqrt{\pi \ell}=K_{C}$, which has not been reported in the literature.

Availability of data: This paper has no additional data.
[1] Hydraulic $\quad$ Fracturing. https

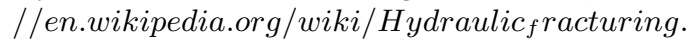

[2] Hydraulic Fracturing - Unlocking America's Natural Gas Resources, American Petroleum Institute, July 2014.

[3] Wen-Hao Shen and Ya-Pu Zhao, Quasi-Static Crack Growth Under Symmetrical Loads in Hydraulic Fracturing, Journal of Applied Mechanics 84(8) (2017). 